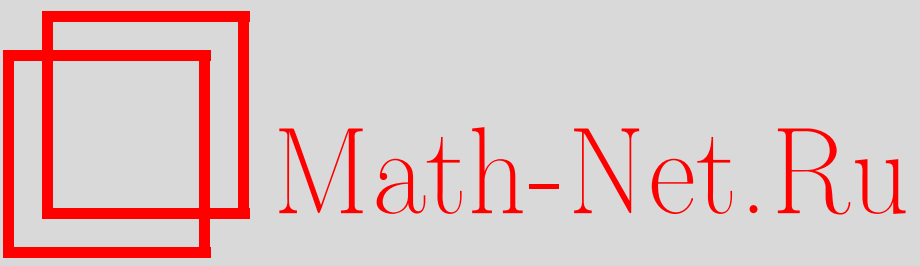

Н. М. Адрианов, О необходимых условиях существования порождающих конечных групп, УМН, 2004, том 59, выпуск 3, 155-156

DOI: https://doi.org/10.4213/rm741

Использование Общероссийского математического портала Math-Net.Ru подразумевает, что вы прочитали и согласны с пользовательским соглашением

http://www.mathnet.ru/rus/agreement

Параметры загрузки:

IP: 52.87 .193 .239

26 апреля 2023 г., 15:14:13 


\title{
О НЕОБХОДИМЫХ УСЛОВИЯХ СУЩЕСТВОВАНИЯ ПОРОЖДАЮЩИХ КОНЕЧНЫХ ГРУПП
}

\author{
Н. М. АдРИАНОВ
}

Пусть $G$-конечная группа, $C_{1}, \ldots, C_{r}$ - некоторьй набор классов сопряженности группы $G$. Существует ли набор порождающих $x_{1}, \ldots, x_{r} \in G$ таких, что

$$
x_{1} \cdots x_{r}=1 \text { и } x_{i} \in C_{i}, i=1, \ldots, r ?
$$

Часто при решении этого вопроса (см., например, [1]) выбирается некоторое представление группы $G$ перестановками, и в качестве необходимого условия применяется теорема Ри ([2], теорема 1 ниже). В настоящей заметке мы предлагаем другое необходимое условие, основанное на линейном представлении группы $G$ в пространстве гомологий $H_{1}(X, \mathbb{C})$ некоторой римановой поверхности $X$. Результат Макбета ([3], теорема 2 ниже) выражает характер этого представления в теоретико-групповых терминах. Мы показываем, что $H_{1}$-условие сильнее условия Ри для любого представления группы $G$ перестановками (теорема 3 ).

В качестве приложения мы приводим (теоремы 4 и 5) список троек классов сопряженности $\left(C_{1}, C_{2}, C_{3}\right)$ групп Матье $\mathbf{M}_{11}$ и $\mathbf{M}_{12}$, для которых не существует порождающих, удовлетворяющих условию (1), что перекрьвает результаты работы [1], касающиеся этих групп.

1. Пусть $G: \Omega$ - действие группы $G$ на $\Omega, c(x)$ - количество независимшіх циклов перестановки $x, c_{i}(x)$ - количество цик лов длины $i, \operatorname{ord}(x)$ - порядок элемента $x, \mathrm{Cl}(x)$ - класс сопряженности элемента $x, N_{G}(\langle x\rangle)$ - нормализатор в $G$ циклической подгруппы, порожденной элементом $x$.

2. Для всякого транзитивного действия $G: \Omega$ и набора порождающих $\left\{x_{1}, \ldots, x_{r}\right\}$, удовлетворяющих условию (1), можно построить компактную связную риманову поверхность $X$ и накрытие $f: X \rightarrow \mathbb{P}^{1}(\mathbb{C})$, неразветвленное вне $\left\{z_{1}, \ldots, z_{r} \mid z_{i} \in \mathbb{P}^{1}(\mathbb{C}), z_{i} \neq z_{j}\right\}$, такие, что группа монодромии $f$ есть $G$, а обходы вокруг точек $z_{i}$ соответствуют порождающим $x_{i}$. Следующий резулттат, по сути, является следствием формулы Римана-Гурвица

Теорема 1 (Ри). Если транзитивная группа перестановок $G: \Omega$ порождена әлементами $x_{i} \in G, i=1, \ldots, r$, такими, что $x_{1} \cdots x_{r}=1$, то

$$
\sum_{i=1}^{r} c\left(x_{i}\right) \leqslant(r-2)|\Omega|+2 .
$$

Если транзитивное действие $G: \Omega$ - регулярное (т.е. $|G|=|\Omega|$ ), то группа $G$ действует на $X$ автоморфизмами. Род римановой поверхности $X$ определяется из формулы Римана-Гурвица: $2 g-2=|G|\left((r-2)-\sum_{i=1}^{r} 1 / m_{i}\right)$. Рассмотрим представление группы $G$ в пространстве $H_{1}(X, \mathbb{C})$. Характер этого представления определяется формулой Лефшеца (см. [4]):

$$
\chi_{H_{1}}(x)= \begin{cases}2-F(x), & \text { если } x \neq 1, \\ 2 g, & \text { если } x=1,\end{cases}
$$

где $F(x)$ - число неподвижных точек автоморфизма $x \neq 1$.

Tеорема 2 (Макбет).

$$
F(x)=\left|N_{G}(\langle x\rangle)\right| \sum_{i=0}^{r} \frac{\varepsilon_{i}(x)}{m_{i}},
$$

где $x \neq 1, m_{i}=\operatorname{ord}\left(x_{i}\right), a \varepsilon_{i}(x)=1$, если $x$ сопряжен какому-нибудь әлементу из $\left\langle x_{i}\right\rangle$, и 0 иначе.

$H_{1}$-УСЛОвиЕ. Пусть группа $G$ порождена әлементами $x_{1}, \ldots, x_{r}$, удовлетворяющими условию (1), а функиия $\chi_{H_{1}}$ задана формулами (3) и (4). Тогда для любого неприводимого характера $\chi$ группь $G$ скалярное произведение $\left(\chi_{H_{1}} \mid \chi\right)$ есть неотрицательное челое число.

ТеОрема 3. Если выполнено $H_{1}$-условие, то для любого транзитивного действия $G: \Omega$ выполнено условие $P$ и (2).

Лемма. Для транзитивной зруппь перестановок $G: \Omega$ верно следующее:

(a) $c(x)=\frac{1}{m} \sum_{k=1}^{m} c_{1}\left(x^{k}\right)$, где $m=\operatorname{ord}(x)$; 
(b) $c_{1}(x)=\frac{|G| \cdot|\mathrm{Cl}(x) \cap H|}{|H| \cdot|\mathrm{Cl}(x)|}$, где $H \subset G-$ стабилизатор некоторой точки $\omega \in \Omega$;

(c) $\sum_{x \in G} c_{1}(x)=|G|$;

(d) $\frac{1}{|G|} \sum_{x \neq 1} c_{1}(x) F(x)=\frac{|G|}{|H|} \sum_{i=1}^{r} \frac{1}{m_{i}} \sum_{k=1}^{m_{i}-1} \frac{\left|\mathrm{Cl}\left(x_{i}^{k}\right) \cap H\right|}{\left|\mathrm{Cl}\left(x_{i}^{k}\right)\right|}$.

ДОКАЗАТЕЛЬСТво ТЕОРЕМЫ 3 . Функция $c_{1}(x)$ является перестановочным характером, соответствующим действию $G: \Omega$, поэтому из $H_{1}$-условия следует, что $\left(c_{1} \mid \chi_{H_{1}}\right) \geqslant 0$;

$$
\begin{aligned}
0 & \leqslant\left(c_{1} \mid \chi_{H_{1}}\right)=\frac{1}{|G|} \sum_{x \in G} c_{1}(x) \bar{\chi}_{H_{1}}(x)=\frac{2}{|G|} \sum_{x \in G} c_{1}(x)+\frac{2 g-2}{|G|} c_{1}(1)-\frac{1}{|G|} \sum_{x \neq 1} c_{1}(x) F(x) \\
& =2+\frac{|G|}{|H|}\left(r-2-\sum_{i=1}^{r} \frac{1}{m_{i}}\right)-\frac{|G|}{|H|} \sum_{i=1}^{r} \frac{1}{m_{i}} \sum_{k=1}^{m_{i}-1} \frac{\left|\mathrm{Cl}\left(x_{i}^{k}\right) \cap H\right|}{\left|\mathrm{Cl}\left(x_{i}^{k}\right)\right|}=2+(r-2)|\Omega|-\sum_{i=1}^{r} c\left(x_{i}\right) .
\end{aligned}
$$

3. Проверка $H_{1}$-условия является механической, если известна таблица неприводимых характеров группы $G$. Следующие результаты получены с использованием компьютерной системы GAP. Исползуемые обозначения классов сопряженности совпадают с принятыми в [5].

Tеорема 4 . B группе $G=\mathbf{M}_{11}$ не существует порожддающих, удовлетворяющих условию (1), для следующих троек классов сопряженности:

$$
\begin{array}{llllll}
(2 A, 3 A, 8 A) & (2 A, 3 A, 8 B) & (2 A, 3 A, 11 A) & (2 A, 3 A, 11 B) & (2 A, 4 A, 5 A) & (2 A, 4 A, 6 A) \\
(2 A, 4 A, 8 A) & (2 A, 4 A, 8 B) & (2 A, 5 A, 5 A) & (2 A, 5 A, 6 A) & (2 A, 6 A, 6 A) & (3 A, 3 A, 4 A) \\
(3 A, 3 A, 5 A) & (3 A, 3 A, 6 A) & (3 A, 4 A, 4 A) & (4 A, 4 A, 4 A) . & &
\end{array}
$$

Tеорема 5. B группе $G=\mathbf{M}_{12}$ не существует порождающих, удовлетворяющих условию (1), для следующих троек классов сопряженности:

$\begin{array}{llllll}(2 A, 3 A, 8 A) & (2 A, 3 A, 8 B) & (2 A, 3 A, 10 A) & (2 A, 3 B, 8 A) & (2 A, 3 B, 8 B) & (2 A, 3 B, 10 A) \\ (2 A, 3 B, 11 A) & (2 A, 4 A, 5 A) & (2 A, 4 A, 6 A) & (2 A, 4 A, 6 B) & (2 A, 4 A, 8 A) & (2 A, 4 B, 6 A) \\ (2 A, 4 B, 6 B) & (2 A, 4 B, 8 B) & (2 A, 5 A, 5 A) & (2 A, 5 A, 6 A) & (2 A, 6 A, 6 A) & (2 B, 3 A, 8 A) \\ (2 B, 3 A, 8 B) & (2 B, 3 A, 10 A) & (2 B, 3 A, 11 A) & (2 B, 3 B, 8 A) & (2 B, 3 B, 8 B) & (2 B, 4 A, 5 A) \\ (2 B, 4 A, 6 A) & (2 B, 4 A, 6 B) & (2 B, 4 A, 8 A) & (2 B, 4 A, 8 B) & (2 B, 4 A, 10 A) & (2 B, 4 A, 11 A) \\ (2 B, 4 B, 5 A) & (2 B, 4 B, 6 A) & (2 B, 4 B, 6 B) & (2 B, 4 B, 8 A) & (2 B, 4 B, 8 B) & (2 B, 4 B, 10 A) \\ (2 B, 4 B, 11 A) & (2 B, 5 A, 5 A) & (2 B, 5 A, 6 B) & (2 B, 5 A, 8 A) & (2 B, 5 A, 8 B) & (2 B, 6 B, 6 B) \\ (2 B, 6 B, 8 A) & (2 B, 6 B, 8 B) & (2 B, 8 A, 8 A) & (2 B, 8 B, 8 B) & (3 A, 3 A, 4 A) & (3 A, 3 A, 4 B) \\ (3 A, 3 A, 5 A) & (3 A, 3 A, 6 B) & (3 A, 3 A, 8 A) & (3 A, 3 A, 8 B) & (3 A, 3 B, 4 A) & (3 A, 3 B, 4 B) \\ (3 A, 4 A, 4 A) & (3 A, 4 A, 4 B) & (3 A, 4 A, 5 A) & (3 A, 4 A, 6 B) & (3 A, 4 A, 8 A) & (3 A, 4 B, 4 B) \\ (3 A, 4 B, 5 A) & (3 A, 4 B, 6 B) & (3 A, 4 B, 8 B) & (3 B, 4 A, 4 A) & (3 B, 4 B, 4 B) & (4 A, 4 A, 4 A) \\ (4 A, 4 A, 4 B) & (4 A, 4 A, 5 A) & (4 A, 4 A, 6 B) & (4 A, 4 A, 8 A) & (4 A, 4 A, 4 B) & (4 B, 4 B, 4 B) \\ (4 B, 4 B, 5 A) & (4 B, 4 B, 6 B) & (4 B, 4 B, 8 B) . & & & \end{array}$

\section{СПИСОК ЛИТЕРАТУРЫ}

[1] M. Conder // Bull. London Math. Soc. 1991. V. 23. № 5. P. 445-453. [2] R. Ree // J. Combin. Theory Ser. A. 1971. V. 10. P. 174-175. [3] A. M. Macbeath // Bull. London Math. Soc. 1973. V. 5. № 1. P. 103-108. [4] H. M. Farkas, I. Kra. Riemann Surfaces. New York: SpringerVerlag, 1980. [5] J. H. Conway, R. T. Curtis, S. P. Norton, R. A. Parker, R. A. Wilson. ATLAS of Finite Groups. Eynsham: Oxford Univ. Press, 1985. 